\title{
EL TRATAMIENTO DE LOS MIOMAS UTERINOS (1876-1936). UNA CONTROVERSIA PROFESIONAL ENTRE CIRUJANOS Y RADIÓLOGOS
}

\author{
Rosa María Medina Doménech
}

Los miomas uterinos fueron una de las afecciones más polémicas para la extensión del uso terapéutico de las radiaciones. La abundante literatura médica que generó en la prensa española, sin parangón con ninguna otra enfermedad benigna en las que se ensayó la radioterapia, es buena muestra de ello.

Al tratarse de una enfermedad benigna, tradicionalmente en manos de los cirujanos, la justificación de un remedio nuevo con escasa fundamentación científica hacía más difícil la negociación entre radioterapeutas y ginecólogos a los que se les disputaba el monopolio terapéutico. Aquí la oferta de una nueva mercancía terapéutica no podía ampararse en el carácter mortal de la enfermedad ni en su papel adjuvante a la cirugía, argumentos que habían justificado la introducción de la radioterapia en el caso del cáncer (1).

El estudio de este tipo de controversias presenta el interés de exponer el carácter no privilegiado del conocimiento científico, pues en su elaboración intervienen conflictos intraprofesionales y procedimientos retóricos de negociación (2) (persuasión y consenso) tanto como factores internos. 
Durante la primera década del presente siglo el tratamiento de los miomas fue, básicamente, quirúrgico (3). Desde que en 1876 practicara Winckel (1837-1912) el primer raspado para detener una metritis hemorrágica, numerosos procedimientos paliativos fueron sucediéndose en la práctica de los cirujanos: la castración ovárica, abandonada por la mortalidad ocasionada; las ligaduras extraperitoneales de las arterias uterinas; la opoterapia mamaria, etc. Con posterioridad, y a pesar del cuestionamiento sobre la gravedad de la enfermedad, otros procedimientos con intención curativa gozaron del beneplácito de los cirujanos, la extirpación de miomas vaginales, la miomectomía vaginal o abdominal, la enucleación y, finalmente, la histerectomía total o subtotal (4).

En su proceso de constitución, la radioterapia fue extendiendo sus indicaciones a diversas patologías en las que la intervención quirúrgica estaba contraindicada. De esta forma se dio el primer paso para el ensayo terapéutico de las radiaciones en los miomas inoperables (5). Los portavoces del nuevo remedio hacían una lectura triunfal del hecho, tributo al empeño investigador que había conducido la roentgenterapia profunda "a las cumbres más altas de la terapéutica ginecológica» (aún reconociendo el beneficio de la ineficacia quirúrgica).

Los primeros casos fueron publicados por Deutch y Morton (18451920) en 1902, que obtuvieron una reducción tumoral marcada y una transformación de las menorragias en menstruaciones normales (6). En España las primeras experiencias fueron presentadas, en 1915, por Recaséns (7), al Congreso de Valladolid de la Asociación Española para el Progreso de las Ciencias, y Calatayud Costa (8), al VII Congreso Internacional de Electrología y Radiología, celebrado en Lyón (9). En 1917 el ginecólogo Fargas Raymat (1893-1925) concluía su tesis de doctorado con la aseveración «La roentgenterapia profunda es una medicación utilísima en el tratamiento de los fibromas uterinos» (10) por su valor curativo, paliativo o auxiliar a los métodos cruentos, y la consideraba exclusivamente indicada en las pacientes con más de cuarenta años. Aún sin sentar los criterios que determinaran el grupo específico de pacientes que pudieran beneficiarse, el nuevo remedio generó un gran entusiasmo, hasta el punto "quizá una exageración» (11) de substituir del todo el bisturí por los rayos $\mathrm{X}$ en el tratamiento de los miomas.

En efecto, el limite de la edad, defendido inicialmente, fue sometido a discusión "no sólo por la mayor acometividad del ginecólogo, sino también por las exigencias de las enfermas que en gran mayoría prefieren recurrir a este medio antes de la operación". Las contraindicaciones queda- 
ban limitadas, según algunos autores (12), para aquellos casos asociados a fenómenos agudos - infecciones o retención de orina-, miomas submucosos, pediculados, esfacelados o asociados a carcinoma. Incluso se aceptaba su uso en mujeres muy anémicas con grandes hemorragias. $\mathrm{Pa}$ ra otros, incluido el propio Fargas hijo,

«La única duda entre la terapia profunda o la intervención quirúrgica, salvo en casos muy especiales, sólo es admisible tratándose de mujeres de más de cuarenta años.»

Para Fargas el inconveniente mayor de la terapéutica radiante residía en su alto coste económico, "cuestión de escasa importancia entre la clientela privada», pero no así entre las pacientes de la beneficencia atendidas en dependencias hospitalarias con escasa dotación. La prolongación del tratamiento durante meses no parecía un inconveniente dado que otras afecciones también requerían tratamientos prolongados y no dificultaba el normal desarrollo de la actividad de las enfermas.

Los detractores del procedimiento argumentaban la similitud de la castración radiológica con la quirúrgica, abandonada desde hacía tiempo. Sus defensores, por contrá, señalaban las bases realmente científicas y la inocuidad de la terapéutica. El mecanismo de acción de los rayos $\mathrm{X}$ se explicaba por su efecto sobre la cortical ovárica —el objetivo era obtener la esterilización - y la acción directa sobre los elementos tumorales demostrada por la efectividad del tratamiento en mujeres menopáusicas. Pero el carácter diferenciado de los miomas contradecía la escala de Bergonié (1857-1925)-Tribondeau (1872-1918) que auguraba una acción más efectiva de los rayos $\mathrm{X}$ sobre células escasamente diferenciadas (13). Por tanto, la explicación de la efectividad había que buscarla en la acción sobre células perivasculares embrionarias y sobre el epitelio de las glándulas uterinas. La diferencia en las respuestas obtenidas se especulaba que estuviera relacionada con las tres fases de la acción radioactiva señaladas por Schultz (1872-1913): irritación, parálisis y destrucción celular. De esta forma, las hemorragias observadas al inicio del tratamiento radiante serían una consecuencia de los efectos irritativos, las curaciones aparentes por tratamiento corto o poco intenso se deberían a una acción limitada a la parálisis y la curación definitiva sólo se produciría alcanzada la destrucción celular. El tratamiento solía ocasionar fenómenos climatéricos, involución vaginal, «borrachera roentgeniana» 
y, sobre todo, lesiones cutáneas graves. Pero estos efectos secundarios eran despreciables, según sus defensores, comparados con los riesgos quirúrgicos (14).

Las mejoras en la dosificación, acaecidas durante la segunda década, especialmente la filtración y la irradiación convergente (15), facilitó el trueque de las indicaciones al reducirse los efectos secundarios. Con todo seguían siendo tributarios de cirugía aquellos casos de mioma cuyo tratamiento radióterápico estaba contraindicado (16). Sin embargo, la indicación no estaba definitivamente perfilada. En 1921 calculaba Conill (17) que, de cada tres casos, uno se intervenía quirúrgicamente (18), a lo que se daba una explicación positiva, en términos de la necesaria ayuda o colaboración entre procedimientos curativos, que «nunca [debieran] contrarrestarse». Pese a esta lectura, parece claro que se dirimía un enfrentamiento de responsabilidades profesionales, como muestra el siguiente comentario del radiólogo Pinós (19) en la conferencia impartida en el curso de cirugía abdominal organizado por Corachán en el Hospital de la Santa Cruz y San Pablo (1927):

«¿Cuándo debe irradiarse y cuándo debe operarse un mioma uterino? He aquí una cuestión que aunque parece resuelta dista mucho de ello. La lucha entre cirujanos y radiólogos está todavía en el período álgido y si unos reclaman para sí la casi totalidad de los casos, los otros pretenden curar con métodos incruentos y por consiguiente menos peligrosos la inmensa mayoría de ellos» (20).

El límite de edad a los cuarenta años aún no estaba consensuado. Para la escuela alemana, representada por los «cirujanos ya radiólogos» Seitz (1871-1961) y Wintz (1887-1947), la edad no significaba ningún impedimento, limitándose las contraindicaciones de la radioterapia a miomas poliposos pediculados, casos de diagnóstico dudoso o cuando la intervención estaba indicada por otras patologías asociadas. Pinós consideraba que frente a los casos claramente quirúrgicos (21) o radiológicos (22) existían otros intermedios en los que "solamente el médico encargado del tratamiento podrá resolver en último término ya que la interpretación de la indicación a seguir es muy elástica».

Una vez adoptado el criterio de irradiar, las técnicas empleadas se habían diferenciado en dos métodos defendidos por distintas escuelas y basados en la defensa de dos mecanismos distintos de acción de los ra- 
yos X. A saber, el método alemán, preconizado por Kronig (1863-), Seitz y Wintz, que defendía el efecto de castración radiológica como único responsable de la regresión del mioma y, en consecuencia, la técnica de irradiación adoptada consistía en una dosis única sobre los ovarios (23). El llamado método francés, por su parte, preconizado por Béclère (18591939) y Solomon (1880-1939) (24), defendía la efectividad en función de la acción directa de la radioterapia sobre la fibra muscular patológica. Las pacientes eran tratadas en catorce o dieciséis sesiones semanales, mediante campos que abarcaban el tamaño tumoral y una dosis de 4000 ó 5000 roentgens. Pinós había adoptado un método mixto que intentaba obviar los inconvenientes de cada una de las técnicas anteriores. De una parte los largos tratamientos de la pauta francesa y por otra, las dificultades de determinar una dosis segura para la obtención de la menopausia. La falta de una justificación científica suficiente para establecer la dosis de castración se subsanaba con los prejuicios que sobre los cuerpos de las mujeres caracterizaron los conocimientos ginecológicos del momento

"hemos notado que en las mujeres con marcados caracteres masculinos la castración es más difícil de obtener que en las que tienen un temperamento linfoide» (25) .

Su técnica consistía en la aplicación de campos que abarcasen el tumor y los ovarios - cuatro o seis campos convergentes-, en dos fases de seis sesiones cada una con un intervalo de mes y medio.

Entrada la década de los treinta, la idea de algunos radiólogos sobre el tratamiento roentgenterápico era triunfalista. Así quedaba reflejado en el texto publicado en 1934 por Riera Vaquer (26) «Exitos de la roentgenterapia: los miomas del útero, fibromas y fibromiomas de la matriz y las hemorragias uterinas", donde la defensa de la roentgenterapia estaba sustentada por su experiencia en el Instituto Fisioterápico, la insuficiencia de la farmacoterapia, el elevado riesgo quirúrgico y razones de orden científico técnico - dosimetría, sensibilidad celular a los rayos Roentgen, empleo de tubos adecuados- (27). Pinós reeditó en 1934 su trabajo (28) publicado en el año veintisiete, que reiteraba los criterios de entonces, y concluía defendiendo la capacidad de resolución curativa de los radiólogos en todos los casos, salvo los claramente quirúrgicos. 
No aceptaban los cirujanos la pérdida del monopolio terapéutico sobre los miomas. Ponjoan, del Servicio de Ginecología del Hospital de la Santa Cruz y San Pablo, trazaba un panorama bien diferente:

\begin{abstract}
«Aunque parezca que las indicaciones y tratamiento de los miomas uterinos es un tema agotado, creo más que interesante, indispensable que los ginecólogos lo retomemos abordando el asunto con valentía, haciendo una llamada de alarma contra la innecesaria, contra la elevada cifra de miomatosas castradas por la roentgenterapia que además de haber perdido los atributos de su sexualidad, en un espacio más o menos dilatado, han tenido que correr al ginecólogo para librarse de las complicaciones del mioma que ha continuado su evolución (...) es necesario que los que a diario, por razón de nuestra especialidad, tenemos ocasión de controlar sus efectos lejanos, llamemos la atención de los internistas mal informados por estadísticas incompletas o amañadas y tendenciosas, asegurando $\mathrm{y}$ demostrando que si los rayos $\mathrm{X}$ ocupan un lugar en el tratamiento de los miomas este es de excepción y no de elección" (29).
\end{abstract}

El cirujano restringía el uso de los rayos Roentgen a los miomas intersticiales no muy voluminosos y en edades próximas a la menopausia. Sin embargo, esta restricción era privativa de la roentgenterapia, no así del radium, cuya manipulación por los ginecólogos se defendía. Las razones argumentadas para apoyar la preferencia eran la facilidad en el manejo, la seguridad en la dosificación, porque su aplicación —-sondaje, histerometría y dilatación- garantizaban la exactitud del diagnóstico, por el menor peligro de lesionar órganos próximos, por carecer de la fase inicial congestiva de la roentgenterapia, por su acción directa sobre la mucosa sangrante $\mathrm{y}$, por último,

«Porque el radium es más ginecológico, con esta definición queremos significar que siendo el especialista, por su dominio de la técnica de exploración genital, el que posee los elementos de juicio necesarios para valorar los caracteres anatomo-patológicos referentes a situación, conexiones y complicaciones del mioma y concomitancia con otras lesiones genitales, datos que constituyen la guía y norma de elección del tratamiento en cada caso particular, la facilidad de manipulación del radium da al ginecólogo el control de la dirección técnica del tratamiento fisiote- rápico de los miomas, prescindiendo de los conocimientos electro-físicos 
indispensables para el manejo escrupuloso de la terapéutica Roentgen» (30).

Como el propio Ponjoan reconocía era el control de la dirección técnica del tratamiento el que decantaba la balanza a favor del radium. No eran necesarias, en esta ocasión, mayores argumentaciones científicas sobre el mecanismo de acción de las radiaciones.

\section{NOTAS}

(1) Una discusión más amplia sobre el desarrollo de la Radioterapia española entendida como el desarrollo de un monopolio profesional puede consultarse en MEDINA DomÉNECH, R. M. (1993), Estrategias profesionalizadoras en el origen de una especialidad médica. El caso de la Radioterapia española (1895-1936). Tesis de Doctorado, Granada (microfichas).

(2) Schuster J. A., Yeo R. R. (1986), The politics and rethoric of scientific method, Dordecht. Gross A. G. (1990), The Rethoric of Science, Cambridge, Mass.

(3) Roselló Gómez, A. (1904), Estudio comparativo de los diferentes tratamientos del mioma uterino. Tesis del doctorado, Barcelona.

(4) Fargas Raymat, M. (1917), «Roentgenterapia en el tratamiento de los miomas del útero", Therapia, 9, 129-57; 193-231, p.130.

(5) Momento denominado como "período caótico de la radioterapia». ConiLl, V. (1916), «Radioterapia ginecológica. Estadística», Therapia, 8, 532-50, p. 544.

(6) Fargas Raymat (1917), pp. 131, 194 y 196.

(7) Sebastián RECASÉns (1863-1933). Entre 1903 y 1933 ocupó la Cátedra de Obstetricia y Ginecología del Hospital Clínico de Madrid, enfrentándose a las aspiraciones de Calatayud de consolidar la Cátedra de Electro-Radiología. Conocido por su tendencia intervencionista, tras el Congreso Ginecológico Internacional de Hạlle (1913), comenzó a defender el uso del radium en neoplasias ginecológicas, iniciando su campaña a favor de la radioterapia en Ginecología, cuestión que generó una amplia polémica con el grupo de cirujanos encabezado por Pulido. Viajó a los Institutos Curie y Roentgen para conocer la técnica de aplicación. En 1923 inauguró unas instalaciones de radioterapia en la Cátedra de Ginecología de Madrid. En 1929 presidía la Junta organizadora de la Liga Nacional Contra el Cáncer. Miguel Velasco, J. E. (1982), Vida y obra de Sebastián Recaséns. Tesis de Doctorado, Madrid.

(8) Celedonio Calatayud Costa (1880-1931). Se doctoró en 1903 con una tesis sobre la roentgenterapia en los fibromas uterinos; un año más tarde, realizó las primeras experiencias con radium en España y en 1905 fundó, en Valencia, el Instituto de Electricidad Médica. Fue fundador de la Revista Española de Electrología y Radiología Médicas (1912). En 1917 era el presidente de la Sociedad Española de Electrología y Radiología Médicas. 
En 1918 obtuvo la primera cátedra del doctorado de Electrología y Radiología en la Facultad de Madrid. Progresos de la Clínica, 18, 18; Medicina Ibera, 17, CXIX.

(9) En ambas comunicaciones afirmaban haber obtenido con las radiaciones la reducción del tumor en más del 50\% de los casos. Fargas RaYmat (1917), p. 202.

(10) Las conclusiones de la tesis, Roentgenterapia en el tractament dels miomas de l'uter, dirigida por su padre Miguel Angel Fargas Roca, aparecieron publicadas en el artículo que reseñamos.

(11) Ibid.

(12) Fargas recogía, entre otras, la opinión de Recaséns.

(13) Bergoníe, J.; Tribondeau, L. (1904 y 1905), "Action des rayons sur le testicule du rat blanc», Seánc. Soc. Biol., 57, 522; 58, 156.

(14) FaRgas Raymat (1917), p. 203.

(15) Consistía en la utilización de múltiples puertas de entrada, incluida la vaginal, para evitar la sobredosificación de zonas cutáneas.

(16) Conill (1916), p. 533, citaba que esta táctica era usual en las clínicas ginecológicas alemanas. Estaba contraindicada la irradiación de miomas muy voluminosos, esfacelados o degenerados.

(17) Víctor Conill Montobbio se formó como ginecólogo con Recaséns en España pero realizó estudios con Wintz y Döderlein en Alemania. Incorporó con rapidez la nueva terapia con radiaciones adquiriendo un aparato Siemens de rayos $\mathrm{X}$ para su consultorio barcelonés. También desempeñó su labor en la Clínica Ginecológica del Hospital Clínico de Barcelona iniciando la radioterapia ginecológica en 1914. Fue vocal de la Junta de la Lucha Contra el Cáncer en Cataluña en 1936. BarRau Vernia, E. J. (1983), El ginecólogo profesor Dr. D. Víctor Conill Montobbio: un humanista. Resumen de la tesis doctoral, Barcelona.

(18) Conill, V. (1921), Indicaciones terapéuticas en el fibromioma uterino. En base a 84 casos operados y 200 tratados por radioterapia profunda, Barcelona, pp. 13-4.

(19) Tomás Angel Pinós Marsell participó en los comités editores de la Revista de Diagnóstico y Tratamiento Físicos (1930) y Medicina Física y fue vicepresidente de la Sociedad de Radiología y Electrología de Cataluña (1933).

(20) Pinós Marsell, T. A. (1927), "Cuándo debe operarse y cuándo debe irradiarse un mioma uterino", Anales del Hospital de la Santa Cruz y San Pablo, 1, 305-8, p. 307.

(21) Miomas degenerados, asociados a patologías en sí mismas quirúrgicas, pediculados, subserosos o submucosos y los escafelados.

(22) Las enfermas muy anémicas, con enfermedades orgánicas o infección externa.

(23) La dosis se estimaba en un 30\% de la dosis eritema y los campos de irradición tenían una gran variabilidad. p. 306 .

(24) Aunque inicialmente ideado por el alemán Albert Schoenberg. PINós (1927),

(25) Ibid., p. 307. Sobre la contribución del discurso médico español del primer tercio del siglo a la definición de la naturaleza femenina puede consultarse ORTIZ, T. (1993), «El discurso médico español sobre las mujeres en la España del primer tercio del siglo veinte», in: LóPEZ BeLtrán, M. T. (ed.), Las mujerés en Andalucía. Actas del 2. ${ }^{\circ}$ Encuentro intedisciplinar de estudios de la mujer en Andalucía, Málaga, pp. 107-138.

(26) Desde su doctorado en 1920, obtenido con un trabajo sobre la ionización en electro-medicina, Joan Riera Vaquer orientó su práctica a la Electrología y Radiología 
Médicas. En 1922 era propietario de una clínica radiológica y miembro de la Sociedad española de Radiología y Electrología Médicas.

(27) Así quedaba reflejado en una reseña aparecida en Archivos del Instituto de Medicina Práctica, 11, p. 223.

(28) PINOS MARSEll, T.A. (1934), "Quan ha d'operarse i quan ha d'irradiarse un mioma uteri», Medicina Física, 1, 5-10.

(29) Ponjoan Sabater, A. (1934), «El tractament d'elecció dels miomas uterins», Annals de l'Hospital de la Santa Creu Sant Pau, 8, 194-8, p. 194.

(30) Ibid., p. 197. 